\title{
Review
}

\section{Nano-scaled Biomolecular Field-Effect Transistors: Prototypes and Evaluations}

\author{
Giuseppe Maruccio $^{* \mathrm{a}, \mathrm{b}}$ Paolo Visconti $^{\mathrm{a}}$, Adriana Biasco ${ }^{\mathrm{a}}$, Alessandro Bramanti ${ }^{\mathrm{a}}$, Antonio Della Torre ${ }^{\mathrm{a}}$ Pier Paolo Pompa $^{\mathrm{a}}$, \\ Vanessa Frascerra ${ }^{\mathrm{a}}$, Valentina Arima ${ }^{\mathrm{a}}$,Eliana D'Amone ${ }^{\mathrm{a}}$, Roberto Cingolani $^{\mathrm{a}}$, Ross Rinaldi ${ }^{\mathrm{a}}$ \\ a National Nanotechnology Laboratory of INFM, University of Lecce, Via per Arnesano, 73100 Lecce, Italy \\ *e-mail: giuseppe.maruccio@unile.it \\ b also at: Department of Physics, University of Lecce
}

Received: March 13, 2004

Final version: April 29, 2004

\begin{abstract}
As modern electronics rapidly approach the ultimate level of integration (typically thought to be at the nanoscale level), the fascinating world of biomolecules provides new opportunities and directions for further miniaturization. In this work we review our results in the field of biomolecular electronics, starting from the fabrication of nanojunctions up to the implementation of hybrid devices.
\end{abstract}

Keywords: Molecular electronics, Nanotechnology, Nanoscience, Downsizing, Bottom-up

\section{Introduction - Miniaturization and Molecular Electronics}

Due to their success, electronics is usually identified with metal-oxide-semiconductor circuits. The remarkable trend of the semiconductor industry in the integration level during the last decades is described by Moore's Law (Figure 1) and has been driven by the continuous downsizing of the components. Up until now, the shrinking and the density improvements primarily came from the significant advances of the lithographic techniques, which reduced the chip area by a combination of smaller feature size (scaling) and product/process redesign (compaction). Even though this miniaturization trend could continue for another 15-20 years, as the size of components approaches the dimension of atoms or molecules, a turning point and conceptually new devices are needed.

The latest edition of the International Technology Roadmap for Semiconductors [1] (a joint effort of worldwide semiconductor industry associations predicting the main trends in the semiconductor industry spanning 15 years into the future) underlines the pressing need for developing beyond-CMOS devices and post-optical lithographies, together with the necessity to engineer manufacturable interconnection schemes compatible with the new materials and processes recently proposed. In various laboratories

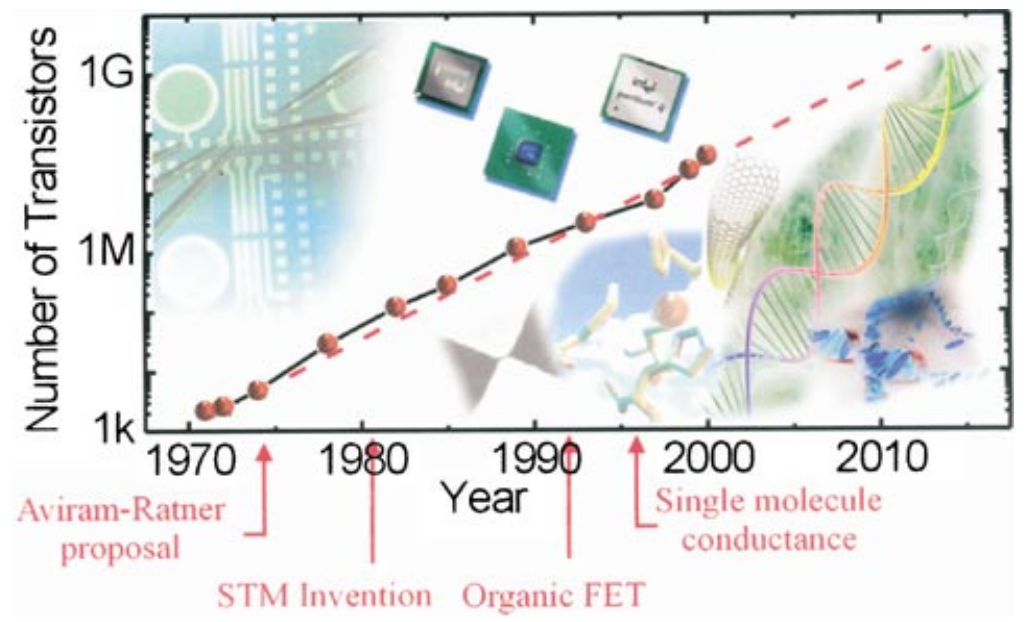

Fig. 1. Moore's law, the 1965 prediction by Intel co-founder Gordon Moore that the number of components per chip would be doubled every 24 months, with resulting declining prices, increasing performance and decreasing cost-per-function (points refer to the various processors introduced by Intel: 4004, 8008, 8080, 8086, 286, 386, 486, Pentium, Pentium II, Pentium III and Pentium 4, respectively). The concomitant occurrence of some milestones of molecular electronics is also indicated. 
minimum feature sizes in the few nanometers range have been demonstrated and extreme ultraviolet [2], X-ray proximity, electron- and ion- beam lithography have been proposed as post-optical techniques. But, to a large extent, these tools are not suitable for mass production and/or capable of meeting the requirements of the Roadmap. So, modern electronics has to face a number of physical and technological limitations as the minimum feature size of a chip is approaching $100 \mathrm{~nm}$ and no known solutions currently exist. Thus, it is becoming increasingly difficult to continue the downscaling, at least with current technologies.

Exploiting a cross-disciplinary bottom-up approach and having the final goal of using interconnected molecules to perform the basic functions of digital electronics, molecular electronics (for a review see ref [3]) has recently attracted much interest as an alternative approach to continue the scaling beyond the $50 \mathrm{~nm}$ node and to maintain the historical trend of reducing the cost/function ratio by ca. $25-30 \%$ / year. The concept of molecular electronics can be traced back to the Aviram-Ratner prediction [4] that molecules having a donor-spacer-acceptor structure would exhibit rectifying properties when placed between two electrodes. Though it needs to merge chemical, biological and physical expertises, the molecular approach offers two very important advantages: the high reproducibility and small size of the molecular building blocks (naturally identical and with well-defined sizes and electronic levels) and their thermodynamically driven self-assembly and self-recognition properties. Recently, worldwide efforts to develop molecularscale electronics succeeded in key demonstrations of individual components [5-11], exploiting the advances both in the fabrication of metallic electrodes with nanometer-scale separation $[12,13]$ and in the synthesis of electronic molecules [14] and the advent of new, very powerful techniques capable of seeing and manipulating single molecules, such as scanning probe microscopy and related techniques. Even though these proofs of concept demonstrate that problems are more technical than conceptual, the integration of circuits at the molecular level remains challenging because of the lack of suitable interconnection schemes for establishing electrical contact between individual molecules. Another great challenge is to move from prototypes to commercial devices, because of the intrinsic difficulty of developing fully-reproducible and high throughput fabrication processes. Moreover, device performance has to be further improved to reach and overtake those of conventional electronic devices.

A very interesting field in molecular electronics is represented by nanobiotechnology, since billions of years of evolution have optimized a large variety of biomolecules (DNA and proteins) capable of performing efficiently a number of different functions. As a consequence, researchers are looking at biology as a source of inspiration, and are working to join biology and nanotechnology. By fusing useful biomolecules to man-made structures, devices may be fabricated that mimic nature in sensing [15], performing complex computational task [16], transferring electrons, relaying, processing and storing information and producing energy [17]. Multifunctional systems [18, 19, 20, 21] and molecular scale motors [22] may also be created. Moreover, the self-assembly and self-recognition properties of biological units open the way to a new generation of devices and fabrication strategies [23]. The concept of self-assembly involves a process known as convergent synthesis and can be defined as the self-organization of one or more entities without any external source of information about the structure to be formed as the total energy of the system is minimized to result in a more stable state. This process inherently implies: a) some mechanism driving movement of entities such as diffusion, electric fields, etc.; b) the concept of "recognition" between different elements, or "bio-linkers", that drives the self assembly to the state of lowest energy.

Important tasks in developing molecular electronics are to study and understand electron transfer between molecules and metal electrodes, to interconnect molecules in real devices and to demonstrate two- and three-terminal molecular devices. In this work, we review our results in the field of biomolecular electronics, starting from the fabrication of nanojunctions up to the implementation of hybrid devices. In Section 2, we discuss how to interconnect molecules in real devices. In Section 3 and 4, we demonstrate two different field-effect transistors based on a modified DNA base and the metalloprotein Azurin. Finally, in Section 5 we will draw some conclusions.

\section{Interconnection of Molecules in Real Devices}

The implementation of molecular electronic devices is based on three fundamental steps:

a. the nanofabrication of a pattern to interconnect molecules to the external world (load, power supply, circuit, etc.), usually following a "top-down" lithographic approach.

b. the delivery of molecules in layers, patterns or to specific locations.

c. the study of molecular electronic properties and their exploitation for device implementation.

In order to fabricate a real device, metals have to be chosen to both create, as far as possible, an ohmic moleculemetal contact (this is a main point still subject of intense experimental and theoretical investigation) and, at the same time, allow the nanofabrication process (for example the lift-off is strongly influenced by the choice of the metal). Gold typically satisfies both these requirements. Moreover, for the implementation of field-effect devices, the choice of the gate oxide is crucial since it determines the leakage currents and limits the maximum gate voltage that can be applied (usually tens of volts).

For a long time, experimental investigations of the fundamental processes involved in electron transfer through molecules have been performed by scanning probe techniques (both scanning tunneling microscopy [24] and conductive probe atomic force microscopy [25]), in which a metallic tip is positioned above molecules deposited on a conducting substrate. Building electronic devices at the 
molecular level is a complicated task due to typical dimensions at nanometer level. Nowadays, with the progress achieved in lithographic techniques, various approaches to interconnect molecules and probe molecular conductivity in real devices (both two- and three-terminal devices) have been demonstrated, including nanopores [26], break junctions [27] and electrodeposited electrodes. Central to all nanofabrication methods is lithography [28], a collective term for several, sometimes very different processes including extreme ultraviolet lithography [2], X-ray proximity lithography, electron- and ion- beam lithography, and soft lithography - followed by further processing like electrodeposition [13] and electromigration.

Of all these options, planar metal-insulator-metal nanojunctions, consisting of two face-to-face metallic electrodes separated by an insulating medium, permit in-plane transport experiments at the surface of a solid (such as on selfassembled molecular layers on functionalized substrates) and the investigation of the dependence of transport mechanisms on the number of molecules interconnecting the electrodes (i.e., on the inter-electrode distance). Moreover, a field-effect device can be easily fabricated by introducing a gate electrode on the substrate. Recently, we demonstrated two different reproducible techniques $[29,30]$ to reduce the separation between two facing EBL-fabricated electrodes to the range of few nanometers: the first one is based on a pre-exposure technique [29], the second exploits electrodeposition [30].

The nanojunctions are made on the oxide surface of thermally oxidized silicon wafers [29] - the thickness of the gate oxide ranges from 100 to $500 \mathrm{~nm}$. Fabrication involves a combination of photolithography and electron beam lithography with the fine features defined in a poly(methyl methacrylate) (PMMA) resist layer. During the first two steps of the process, photolithography followed by lift-off is used to define the $\mathrm{Ag} 50 \mathrm{~nm}$-thick gate electrode on the back of the $\mathrm{Si} / \mathrm{SiO}_{2}$ substrate and the contact pads $(\mathrm{Cr} / \mathrm{Au}, \mathrm{Ti} / \mathrm{Au}$, $\mathrm{Ti} / \mathrm{Pt}$, thicknesses $6 / 60 \mathrm{~nm}$ ) on $\mathrm{SiO}_{2}$. Successively, the EBL process is carried out with a Leica LION LV1 system having a thermal field emission electron source with a $5 \mathrm{~nm}$ pointsize e-beam. All the fabricated nanodevices are inspected by plane-view scanning electron microscopy (SEM) in order to establish the success of the whole process and to estimate the separation between the nanotips. Electrical characterization of empty nanojunctions is performed by $I-V$ measurements using a semiconductor parameter analyzer. Typically, about $90 \%$ of nanojunctions are of good quality with noleakage and open-circuit resistance higher than 100-200 G $\Omega$. Using this standard EBL process, we obtain inter-electrodes separation not smaller than $30-40 \mathrm{~nm}$. The final electrode distance reflects exactly the designed one. Note that the progressive reduction of the electrode width approaching the nanojunction - in a tip-like geometry - is useful in order to reach very small inter-electrode distances, since it facilitates the lift-off process [12].

The inter-electrode separation can be reduced down to few nanometers using a brushing method [29] or by means of an additional process consisting of $\mathrm{Au}$ electrodeposition
[30]. The first method consists in a partial exposure of the resist before the standard EBL process, by brushing the PMMA layer for a precise short time, in the range of few seconds, with a defocused electron-beam. In Figure 2a, the reduction of tip separation as a function of the brushing time is shown. By a careful calibration of the brushing time between 10 and $60 \mathrm{~s}$, the inter-electrode distance is reduced almost linearly from $50 \mathrm{~nm}$ down to $10 \mathrm{~nm}$; for $t>60 \mathrm{~s}$, the tip separation goes to zero. The inset of Figure 2a outlines our exposure technique. Figure $2 b$ shows a high magnification SEM image of $\mathrm{Cr} / \mathrm{Au}$ nanotips with separation of only $20 \mathrm{~nm}$ obtained by EBL with additional e-beam brushing $(t \cong 40 \mathrm{~s})$. Electrode distance could also be reduced by increasing the dose, but we lack a fine control of the process in this case.

In the second approach, an additional Au electrodeposition step is performed by using a commercial Au-cyanide electroplating set-up. High magnification (HM) SEM images of nanojunctions fabricated by Au electrodeposition are reported in Figure 3. The calibration plot of the $\mathrm{Au}$ electrodeposition process (Figure 3a) shows the reduction of inter-electrode gap as a function of process duration, from an initial value of $100 \mathrm{~nm}$, to a resolvable minimum gap of only $7 \pm 2 \mathrm{~nm}$ (Figure $3 \mathrm{~d}$ ). The lateral growth rate varies from $1.66 \mathrm{~nm} / \mathrm{s}$ at the beginning to $2.5 \mathrm{~nm} / \mathrm{s}$ when the gap is reduced below $20 \mathrm{~nm}$. Due to possible small instabilities of electrodeposition process parameters (temperature and $\mathrm{pH}$ of the solution, resistance of the contacts, etc.) with consequent changes of the Au lateral growth rate, the percentage yield for a programmed sub-10-nm gap is in the range $20-30 \%$. To remove all PMMA residues and contaminations between the planar electrodes, an $\mathrm{O}_{2}$ plasma treatment is carried out at the end of this process. Both these methods to reduce the inter-electrode separation present proper advantages and drawbacks. The brushing technique provides a higher throughput while the Au electrodeposition allows to obtain more close electrodes, although in this case contaminations between the planar electrodes reduce the process yield.

\section{Guanosine-Based Field-Effect Transistors}

Recently, the attention of nanotechnologists has focused on biological systems due to the opportunities inherent in their self-assembly and self-recognition properties and the possibility to exploit systems engineered by nature as functional components in devices. Two different pathways to selfassemble biological units on a suitable substrate are surface absorption (physisorbtion) and covalent binding at defined sites, taking advantage of the specific reactivity of molecules having functional groups with affinity for specific surface atoms and/or molecular sites. Recently we demonstrated two different biomolecular field-effect transistors that exploit the self-organization in the solid-state of a modified DNA base (called deoxiguanosine [6]) and the covalent bonding of a metalloprotein (Azurin), onto planar nanoelectrodes [31]. 

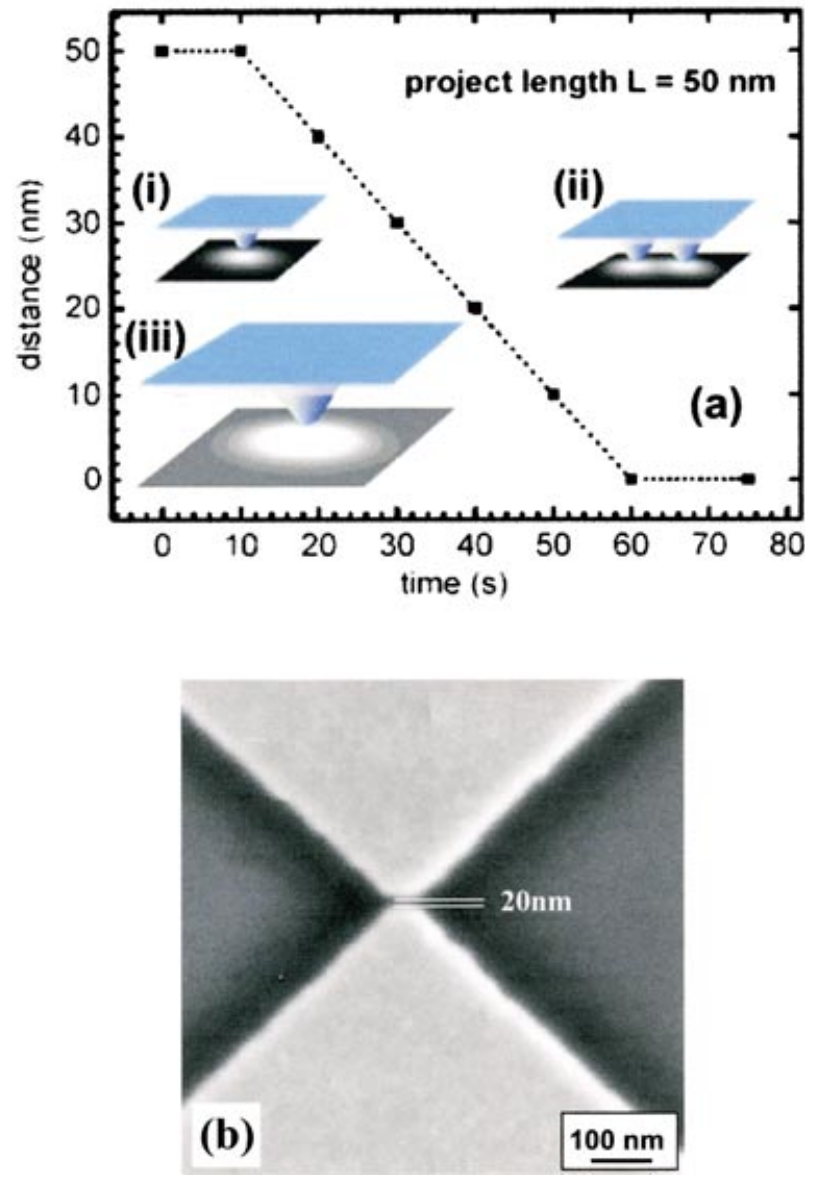

Fig. 2. a) Reduction of the separation between electrodes as a function of the brushing time $(\Delta t)$ of PMMA resist by a defocused e-beam. When $\Delta t$ ranges from 0 to $50 \mathrm{~s}$, the tip separation is reduced almost linearly from $50 \mathrm{~nm}$ (the nominal one) to $10 \mathrm{~nm}$; for $\Delta t=60 \mathrm{~s}$, the tip separation becomes zero. The inset outlines our exposure technique. (i) Gaussian shape of the e-beam; (ii) The exposure overlap between two adjacent points. (iii) Our exposure strategy. First, the resist is partially exposed by means of a defocused e-beam (gray background in the inset, instead of the previous black one). Then, during the following EBL process, the Gaussian shape of the e-beam allows the resist near to the edges of the tip to reach the right dose, resulting in a final electrode distance $\mathrm{d}$ inferior to the nominal exposure length $L$. The evaluated deviation of $d$ is of the order of $5 \mathrm{~nm}$, due to process parameters such as the point-size of the beam, the step size of exposure and the SEM resolution. b) Plan-view SEM image of $\mathrm{Cr}$ $\mathrm{Au}$ nanotips with separation of $20 \mathrm{~nm}$ obtained with a brushing time of $40 \mathrm{~s}$.

Fig. 3. a) Calibration plot of the $\mathrm{Au}$ electroplating process, showing the reduction of separation between electrodes as function of process duration. b) Cross-section SEM micrograph of electrodes with separation around $20 \mathrm{~nm}$ (sample tilting ca. $60^{\circ}$ ). (c-e) Plan-view SEM images of $\mathrm{Cr} / \mathrm{Au}$ nanotips with tip separations of $15 \mathrm{~nm}, 10 \mathrm{~nm}$, and $7 \mathrm{~nm}$, respectively.
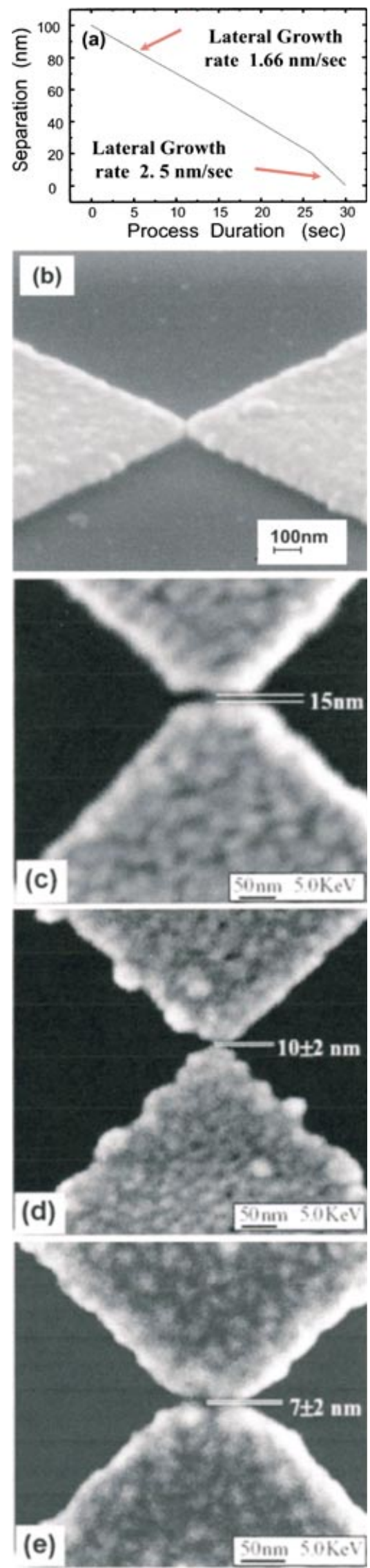

(c) 2004 WILEY-VCH Verlag GmbH \& Co. KGaA, Weinheim 
Unlike other molecular electronic devices recently reported - primarily through the use of carbon nanotubes [9, 10] and polymers [32] - our DNA-based field-effect transistor (FET) involves transport through ordered and self-assembled layers, instead of disordered ones (usually obtained by evaporation or spin-coating) or single molecules with serious interconnection problems (such as in devices based on carbon nanotubes). Recently, large efforts were focused, in various laboratories worldwide, on assessing the electrical transport in DNA strands. In our work, we adopt a different strategy, by choosing a single modified DNA base (a lipophilic deoxyguanosine) as the active component of a field-effect transistor, due to the guanine low oxidation potential, which enhances charge transport. The base is modified, in a lipophilic deoxyguanosine derivative form [33] (Figure 4), to favor the formation of ordered supramolecular structures, thanks to its peculiar sequence of H-bond donor and acceptor groups and side chains. The nanodevices are fabricated by cast deposition of a $2 \mu \mathrm{L}$ drop of deoxyguanosine solution in chloroform $\left(10^{-1}-10^{-4} \mathrm{M}\right)$ in the gap between two $\mathrm{Cr} / \mathrm{Au}$ planar nanoelectrodes fabricated by EBL and with tip separation ranging from 20 to $100 \mathrm{~nm}$ (with the closest electrodes obtained using the brushing technique). The thickness of the $\mathrm{SiO}_{2}$ layer is $100 \mathrm{~nm}$ and the gate electrode is a silver contact on the conductive side of the $\mathrm{Si} / \mathrm{SiO}_{2}$ substrate. Upon controlled evaporation, the deoxyguanosine molecules selfassemble in supramolecular ribbon-like structures as demonstrated by atomic force microscopy (AFM) experiments (Figure 4). Electrical characterization is focused on DC conductivity. Transport experiments are carried out in the voltage range between -3.5 and $3.5 \mathrm{~V}$, at room temperature and ambient pressure. The gate and drain voltage are applied with respect to the grounded electrode (source) and drain-source and gate current are recorded simultaneously. Typical $I-V$ curves under forward bias for five different gate bias are reported in Figure 5. Negligible leakage current is observed (values as low as few pA), demonstrating the good insulation between the planar electrodes and the back gate. All the measured characteristics show the existence of a well-defined threshold voltage and indicate that conduction can be activated at higher bias. Guanosine ribbons behave like an insulator at low voltages, exhibiting a low current in the current voltage characteristics (resistance of tens of $\mathrm{G} \Omega$ ). Current rises following an almost exponential dependence above the threshold voltage $V_{\mathrm{T}} \approx 3 \mathrm{~V}$. No saturation in the current is observed for these voltages. The threshold voltage $\left(V_{\mathrm{T}}\right)$ can be modulated by means of a control gate voltage $V_{\mathrm{g}}$ : by increasing the gate voltage from $0 \mathrm{~V}$ to $1 \mathrm{~V}, V_{\mathrm{T}}$ gradually shifts to higher values following an almost linear trend. A rectification ratio $R R(V)=I(V) / I(-V) \approx 3$ is observed in the entire current-voltage characteristics, suggesting that the intrinsic dipole moment of the single molecule is partially preserved in the supramolecular layer connecting the electrodes, and induces an asymmetry in the charge pathway, resulting in a preferential direction for the current flow. The current at fixed drain-source voltage decreases with $V_{\mathrm{g}}$ (inset of Figure 5, left hand scale) as in a p- channel MOSFET. Transport characteristics could be explained by means of a resonant tunneling effect in the molecular layer when Fermi levels in the metallic contacts align to molecular layer levels. By changing the gate voltage, the alignment condition can be modulated since the molecular band shifts in energy and an additional $V_{\mathrm{T}}$ is needed in order to activate transport across the device [6]. The maximum voltage gain is 0.76 and represents a reasonably good result, when compared to other smallchannel devices $[9,10]$. An on-off ratio around 5 could be enough for some logic applications, such as static random access memory (SRAM). However, the potential barriers at the interface, due to the difficulty in creating an ideal ohmic molecule-metal contact, reduce the device performances $[34,9]$. These features make this device promising for the development of molecular electronic circuits operating at room temperature and ambient pressure. The small size of the device - on the nanometer scale - could allow a large integration at low cost.

\section{Azurin-Based Field-Effect Transistors}

In the second approach we have used the blue-copper protein azurin immobilized in ordered monolayers azurin [8] $(\mathrm{Az})$ is a small electron-transfer and soluble metalloprotein involved in the respiratory phosphorylation of the bacterium Pseudomonas aeruginosa. Its secondary structure and also the presence of the disulfide bond make $\mathrm{Az}$ a very stable protein. Moreover, given its capability to function as a one-electron carrier in the biological environment (due to the equilibrium between the two stable oxidation states of the $\mathrm{Cu}$ ion and to the structural stability of the active site), azurin is very intriguing for molecular electronics. The unique disulfide bridge (Cys3 - Cys26) located at one end of the $\beta$-barrel structured protein and at a distance of $\approx 2.6 \mathrm{~nm}$ from the copper site allows the chemisorption of proteins in oriented monolayers on crystalline gold or on suitably functionalized surfaces. Note that the immobilization procedure is a particularly demanding aspect since it should insure an appropriate and permanent localization of the biomolecules while preserving its function. The adsorption of a protein onto a solid surface is a complex process, including two steps. Translating by Brownian motion, the protein firstly collides with the surface, where it remains if the thermal energy of the protein molecule is smaller than the linking energy. Once the protein is adsorbed, the second step is assumed to involve a conformational change to an equilibrium configuration, by means of local interactions. It is thought that this process could lead to denaturation, and thus to a loss of protein functionality [35]. We developed two different surface chemical strategies to immobilize azurins in ordered monolayers both on gold and $\mathrm{Si} / \mathrm{SiO}_{2}$ substrates, taking advantage of the disulfide bridge to guarantee an unambiguous orientation of the protein.

Direct site-specific attachment on freshly prepared $\mathrm{Au}(111)$ grown on muscovite mica sheets is achieved by incubating them in $70 \mu \mathrm{M}$ azurin $(50 \mathrm{mM}$ sodium acetate, $\mathrm{pH}$ 


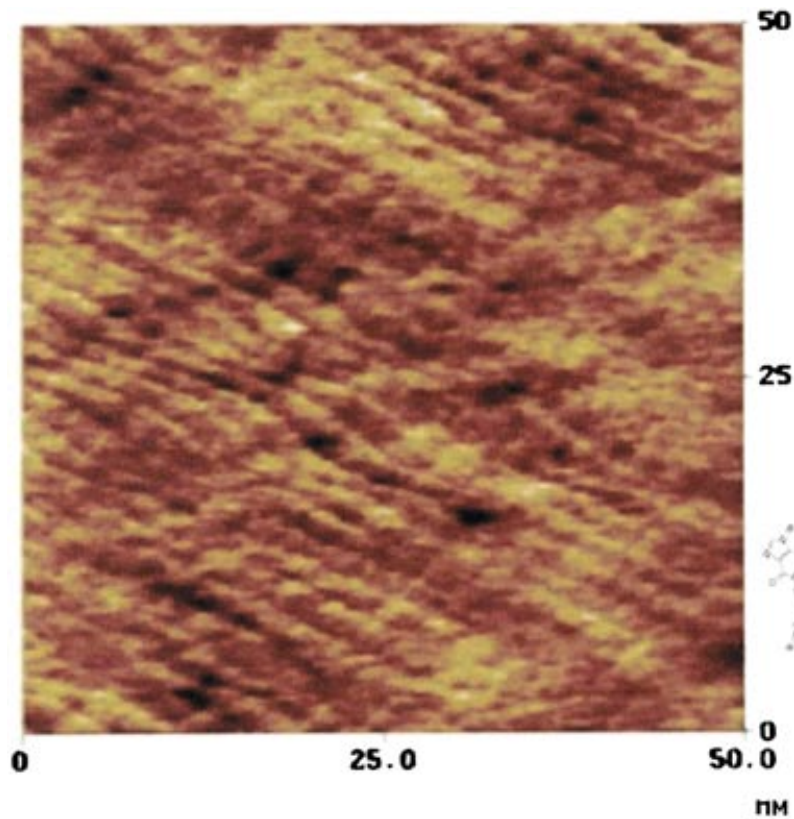

50.0

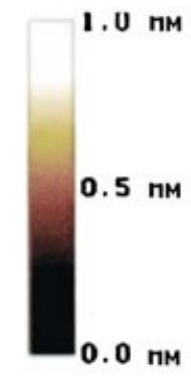

25.0

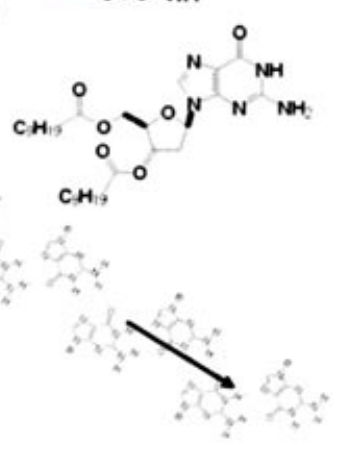

Fig. 4. AFM image of a guanosine layer. The ribbons form an ordered supramolecular structure, laying parallel to each other with a periodicity of $2.5 \mathrm{~nm}$ and a length up to $100 \mathrm{~nm}$ and giving rise to an orthorhombic unit cell of size $a=1.3 \pm 0.1 \mathrm{~nm}$ and $b=2.5 \pm 0.2 \mathrm{~nm}$. For distances longer than $100 \mathrm{~nm}$, that ordering is lost, and the ribbons are randomly oriented.

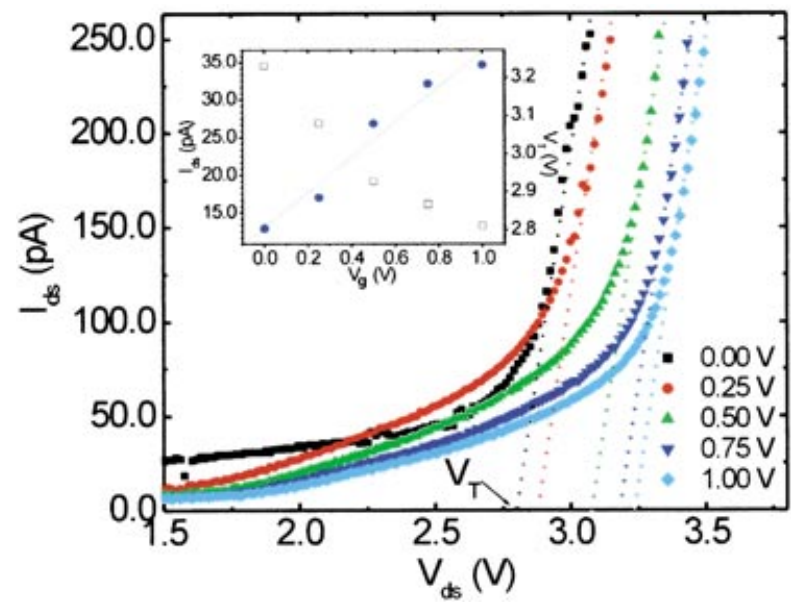

Fig. 5. Characteristics of the dG-based FET at different gate voltages $\left(V_{\mathrm{g}}\right)$. The dashed lines extrapolate the voltage threshold $V_{\mathrm{T}}$ for any $V_{\mathrm{g}}$ value. By changing the gate-voltage, $V_{\mathrm{T}}$ can be modulated since the alignment condition for resonant tunneling is modified due to a shift in the energy of the molecular band. Inset. Left scale: dependence of the drain-source current $\left(I_{\mathrm{ds}}\right.$, hollow squares) at $V_{\mathrm{ds}}=2.0 \mathrm{~V}$ on the gate voltage $V_{\mathrm{g}}$. Right scale: dependence of $V_{\mathrm{T}}$ (circles) on the gate voltage $V_{\mathrm{g}}$.

4.6) at $4{ }^{\circ} \mathrm{C}$ for $1.5 \mathrm{~h}$. Subsequently, samples are gently rinsed with ultrapure water, dried with a soft jet of pure nitrogen and investigated by scanning tunnelling microscopy. Figure 6a reports an STM image acquired in air at a bias voltage of $-700 \mathrm{mV}$ and a current setpoint of $1 \mathrm{nA}$. On the gold terraces, bright spots are clearly visible with a lateral size around $4-6 \mathrm{~nm}$ in good agreement with the reported crystallographic data on azurin dimensions [36]. The surface density of the spots can be tuned by varying incubation time, and protein concentration. The vertical size of the protein extracted by STM data (Figure 6b) is smaller than the crystallographic dimension, as usual for protein adsorbed onto gold and imaged by STM $[37,38]$. In the case of the $\mathrm{SiO}_{2}$ substrates, the technique for protein immobilization is based upon the self assembly of 3-mercaptopropyltrimethoxysilane (3-MPTS). Protein immobilization takes place via the reaction of the free thiol groups of 3-MPTS with the surface disulfide bridge of Az. Thus, the orientation of the molecule is expected to be well-defined, due to the unique disulfide bridge in the protein. Non-contact atomic force microscopy (NC-AFM) was used to image azurin monolayers in order to considerably reduce the force applied between the tip and the protein. A typical image is displayed in Figure 6c. The surface is totally covered by recognizable granular structures, which are not present on silanized substrates. Due to the intrinsic loss of resolution normally introduced in AFMimages by probe tip geometry, the morphological characterization of the azurin monolayers in this case is performed by a height distribution analysis. AFM data are fitted iteratively using a combination of Gaussian functions. The center of the Gaussian function is taken as the defining parameter for molecular height [35] in order to gain information about the three dimensional size and the structural stability of the proteins in dry monolayers. Each height distribution is the average of 5-10 independent AFM images (scan size: $2 \mu \mathrm{m} \times 2 \mu \mathrm{m}$ ) and consists of the convolution of three different terms (Figure 6d) with the dominant contribution (approximately $85 \%$ ) centered around $4 \mathrm{~nm}$, again in general agreement with the crystallographic size of azurin 
[36]. This demonstrates that the chemisorption process does not induce gross denaturation and/or conformational transitions in azurin, which is very important for possible application in the fields of bioelectronics and biosensors. An additional experiment performed on monolayers of intentionally denaturated azurin (thermal unfolding, $1 \mathrm{~min}$ at $80^{\circ} \mathrm{C}$, before immobilization) supports this conclusion, demonstrating that the peak due to the intact azurin monomer becomes broader while the additional peaks ascribed to the formation of aggregates and the structure compaction of proteins - become significantly more prominent (Figure 6e).

To further support the integrity of proteins in dry monolayers, their conformational properties are investigated by intrinsic fluorescence spectroscopy, as the fluorescence of the aromatic residues strongly depends on the protein microenvironment [39]. A tryptophan residue (Trp48) is responsible for protein emission [40, 41]. Since the fluorescence of the copper azurin is strongly quenched by the presence of the copper atom, we utilize the apo-form for optical investigation. The fluorescence spectra of apoazurin monolayers and free proteins in buffer (Figure 7) are very similar and only a slight redshift (ca. $2-3 \mathrm{~nm}$ ) is observed. As a reference, the red-shifted emission spectrum of a denatured sample of azurin [42] is also reported in Figure 7. Thus, we conclude that the covalent binding to the functionalized $\mathrm{SiO}_{2}$ surface does not interfere with the fold pattern of the native protein.

To implement protein devices, nanoelectrodes are fabricated by electron beam lithography in a field-effect transistor configuration, as previously described. The inter-electrode distance and the oxide thickness are both $100 \mathrm{~nm}$. Electrical characterization is focused on DC conductivity at room temperature and ambient pressure. The 3D plot of the drain-source current as a function of the drain source bias $\left(V_{\mathrm{ds}}\right)$ and of the gate bias $\left(V_{\mathrm{g}}\right)$ is reported in Figure 8. While azurin monolayers behave as insulator at low bias, current successively rises up to hundreds of pA at higher voltages. A pronounced resonance centered at $V_{\mathrm{g}}=$ $1.25 \mathrm{~V}$ is observed in the transfer characteristics with transconductance changing from positive to negative values and transistor behavior switching from n-MOS to p-MOS type. The resonance exhibits a peak to valley ratio and a FWHM of 2 and $0.3 \mathrm{~V}$ respectively and gradually disappears
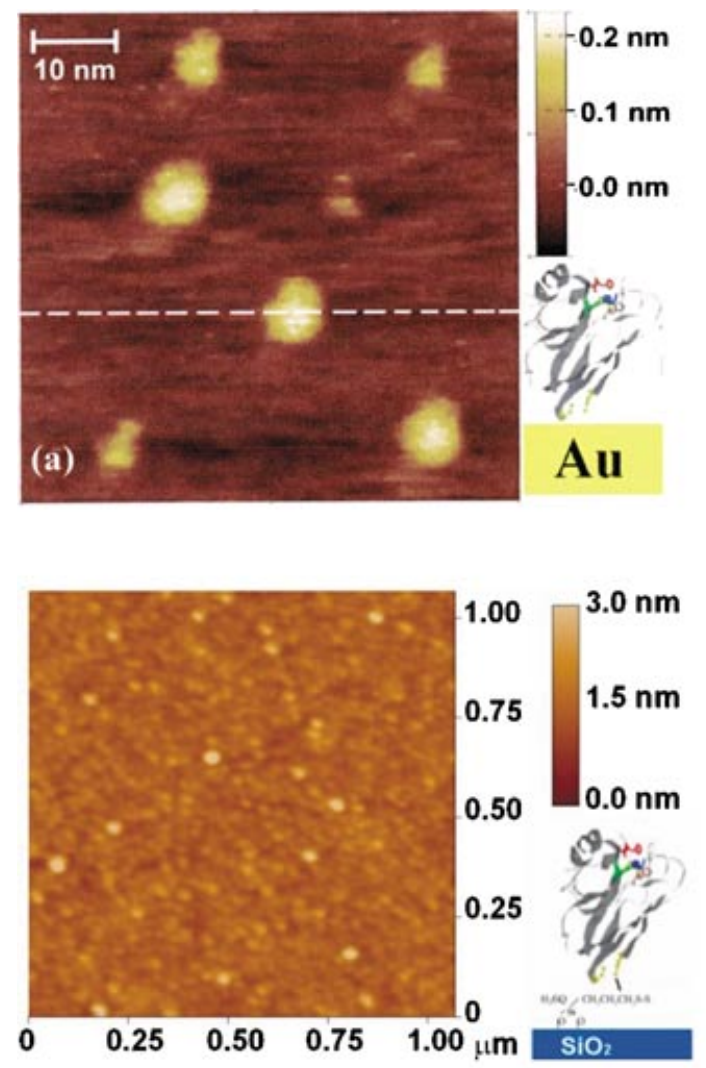
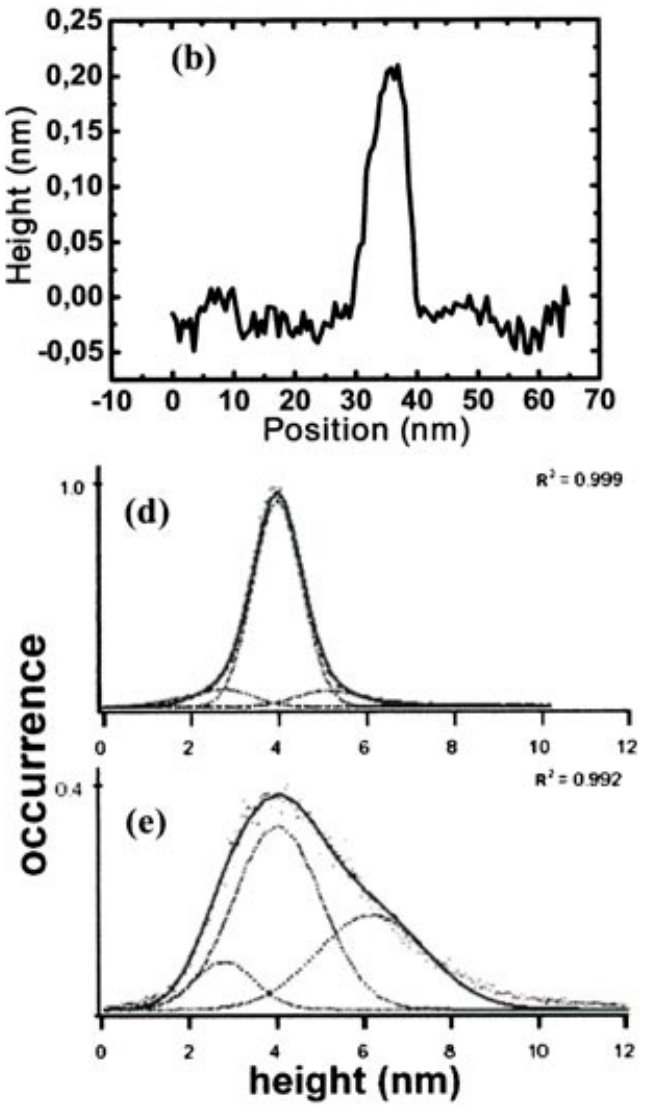

Fig. 6. a) STM topographic image of azurin adsorbed on $\mathrm{Au}(111)$ acquired in constant-current mode in air. The proteins are clearly visible as bright spots. b) Height profile of azurin imaged by STM. The lateral size (FWHM) around 4-6 nm is in good agreement with the reported crystallographic data. c) NC-AFM image of an azurin monolayer self-assembled on functionalized $\mathrm{SiO}_{2}$. Due to the intrinsic loss of resolution introduced by the convolution with the tip geometry, the morphological characterization of these monolayers is performed by a height distribution analysis (d). e) The same analysis performed on monolayers intentionally subjected to a destructive treatment $\left(1 \mathrm{~min}\right.$ at $\left.80^{\circ} \mathrm{C}\right)$. 


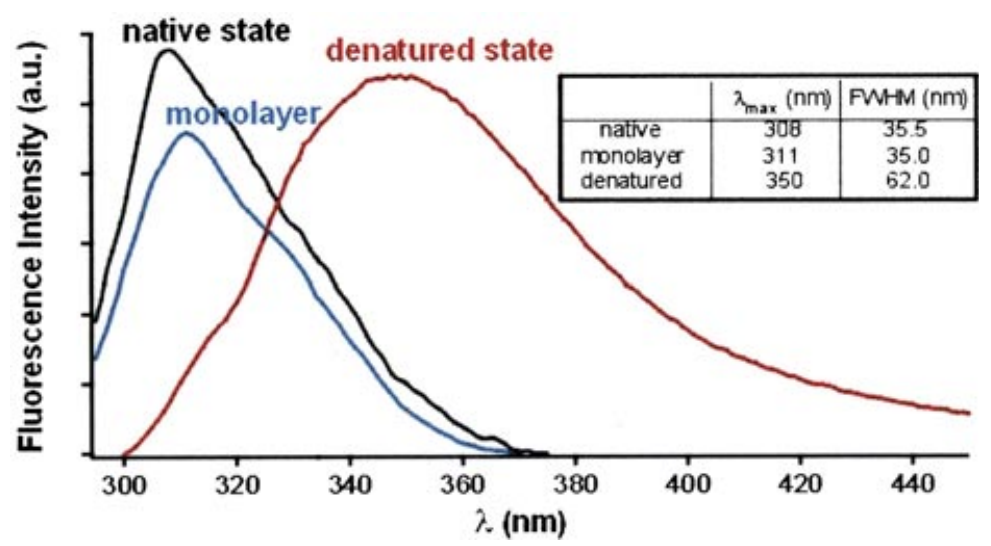

Fig. 7. Fluorescence spectra of free native apoazurin, denatured apoazurin and apoazurin chemisorbed on $\mathrm{SiO}_{2}$.

after some cycles of measurement due to the ageing of the nanojunctions (Figure 9) when high voltage is applied. This peculiar conductivity is ascribed to transport via electron hopping from one reduced $(\mathrm{Cu}(\mathrm{I}))$ molecule to an adjacent oxidized ( $\mathrm{Cu}(\mathrm{II}))$ molecule [43]. Therefore, two adjacent Az molecules with different redox states are required for charge transport. Since the protein is chemisorbed onto the $\mathrm{SiO}_{2}$ surface with the axis joining the copper site to the disulfidebridge almost perpendicular to the surface, the main effect of the gate voltage is to change the oxidation state of the copper site. Consequently, a gate voltage exists where the current is maximum, as the populations of reduced and oxidized proteins become equal; otherwise the current is lower. Recent results of STM experiments performed on azurins chemiosorbed on $\mathrm{Au}(111)$ substrates support this model [44].

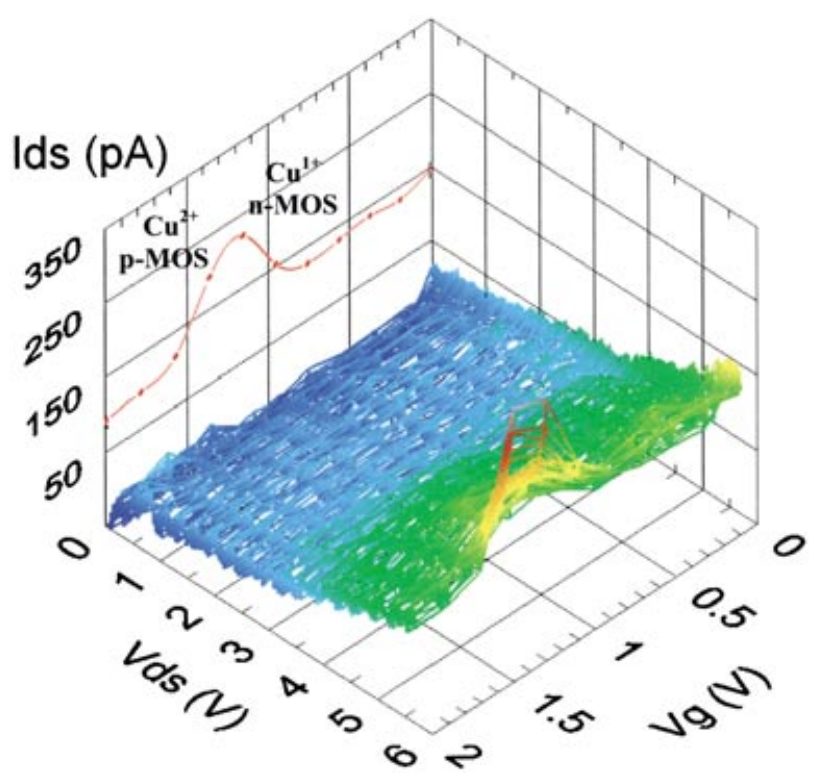

Fig. 8. 3D plot of the drain-source current as a function of the drain-source bias and the gate bias.

\section{Conclusions}

In conclusion, we report two biomolecular FETs exploiting a modified DNA base and the redox and self-assembly properties of the metallo-protein azurin. To complete the discussion, the issues of device ageing and lifetime have to be addressed as a rule for molecular electronics, since the need for high throughput and long-term stability is critical for transferring molecular electronics prototypes to production. Device failure during operation can be accounted to a degradation of the molecular layer and/or a damage of nanojunctions. Even though our preliminary data on protein fluorescence in an electric field demonstrate that the protein preserves its function under high fields, such fields usually lead to a damage of nanojunctions. We observe two main phenomena concerning device damage: (1) blowing up of nanojunctions (Figure 9a) or (2) the formation of various aggregates on the contacts (Figure 9b). When we apply a bias, a very high electric field arises between the electrodes (Figure 9c), because they are placed few tens of nanometers apart and there is a drastic change in the dielectric constant at the interface between $\mathrm{SiO}_{2}$ and the metal. This very intense electric field is the origin of the device damage. We stress that the tip-geometry, though useful to realize very close electrodes, introduces critical breaking points, related to the regions where the electric field is higher (tips). An improvement can be obtained if discontinuities in the electrode geometry are avoided to prevent field-induced damage. In order to reduce these problems, we have started to fabricate electrodes with a trapezoidal shape (Figure 9d).

\section{Acknowledgements}

Financial support by NNL-INFM and EC through SAMBA project is gratefully acknowledged. 

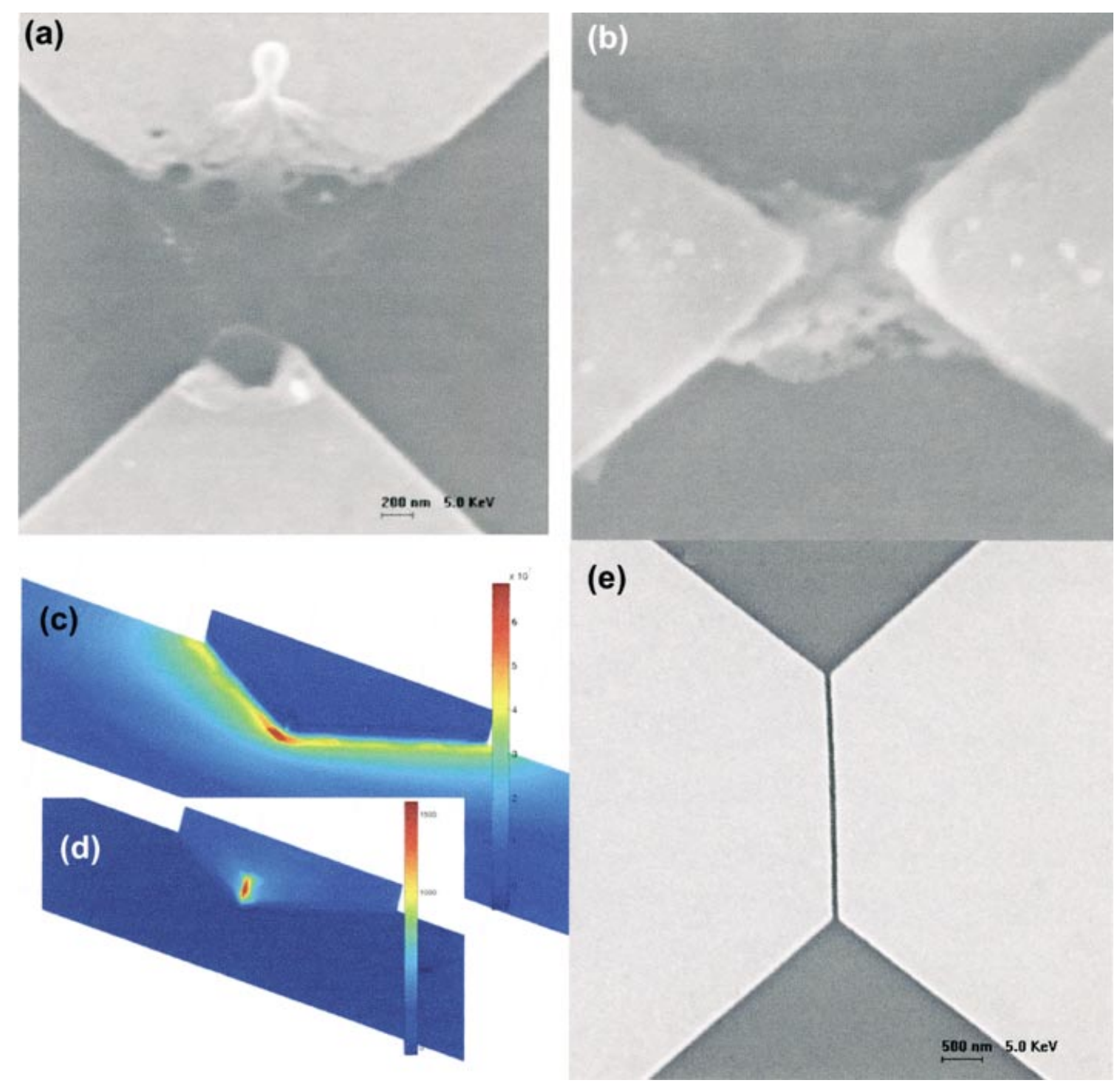

Fig. 9. After the electrical characterization, all devices were inspected by scanning electron microscopy in order to assess their state. We observe two different phenomena: a) nanojunctions blow up with a consequent insulator behaviour; b) the formation of aggregates between the tips, exhibiting an ohmic behavior in the electrical characteristics and probably consisting of metal clusters. These phenomena are related to the high field in the tip region when a bias is applied. Map of the electric field (c) and the current density (d) as result from electromagnetic field simulation using finite elements methods. Electrodes fail where the hotspots in the current density are located, near the final part of the tips. e) To solve this problem, we are now fabricating tips with a different shape, avoiding restrictions in the geometry.

\section{References}

[1] The Internet Technology Roadmap for Semiconductors (ITRS) is available at http://public.itrs.net

[2] C. W. Gwyn, R. Stulen, D. Sweeney, D. Attwood, J. Vac. Sci. Technol. B 1998, 16, 3142 .

[3] G. Maruccio, R. Cingolani, R. Rinaldi, J. Materials Chemistry 2004, 14, 542.

[4] A. Aviram, M. A. Ratner, Chem. Phys. Lett. 1974, 29, 277.

[5] C. Joachim, J. K. Gimzewski, A. Aviram, Nature 2000, 408, 541.

[6] G. Maruccio, P. Visconti, V. Arima, S. D’Amico, A. Biasco, E. D'Amone, R. Cingolani, R. Rinaldi, S. Masiero, T. Giorgi, G. Gottarelli, Nano Lett. 2003, 3, 479.

[7] J. M. Seminario, A. G. Zacarias, J. M. Tour, J. Am. Chem. Soc. 2000, 122, 3015.
[8] M. A. Reed, J. Chen, A. M. Rawlett, D. W. Price, J. M. Tour, Appl.Phys.Lett. 2001, 8, 3735.

[9] J. Appenzeller, J. Knoch, V. Derycke, R. Martel, S. Wind, Ph. Avouris, Phys. Rev. Lett. 2002, 89, 126801.

[10] S. J. Tans, A. R. M. Verschueren, C. Dekker, Nature 1998, $393,49$.

[11] J. Park, A. N. Pasupathy, J. I. Goldsmith, C. Chang, Y. Yaish, J. R. Petta, M. Rinkoski, J. P. Sethna, H. D. Abruna, P. L. McEuen, C. Ralph, Nature 2002, 417, 722.

[12] S. Cholet, C. Joachim, J. P. Martinez, B. Rousset, Eur. Phys. J. AP 1999, 8, 139.

[13] Y. V. Kervennic, H. S. J. Van der Zant, A. F. Morpurgo, L. Gurevich, L. P. Kouwenhoven, Appl. Phys. Lett. 2002, 80, 321.

[14] J. M. Tour, Acc. Chem. Res. 2000, 33, 791.

(c) 2004 WILEY-VCH Verlag GmbH \& Co. KGaA, Weinheim 
[15] L. L. Looger, M. A. Dwyer, J. J. Smith and H. W. Hellinga, Nature 2003, 423, 185.

[16] a) L. M. Adleman, Science 1994, 66, 1021; b) R. S. Braich, N. Chelyapov, C. Johnson, P. W.K. Rothemund, L. Adleman, Science 2002, 296, 499.

[17] For example biofuel cell exploiting enzymes that extract energy from compounds (such as glucose) to power life have been reported: T. Chen, S. Calabrese Barton, G. Binyamin, Z. Gao, Y. Zhang, H. Kim, A. Heller, J. Am. Chem. Soc. 2001, 123, 8630.

[18] N. L. Rosi, J. Eckert, M. Eddaoudi, D. T. Vodak, J. Kim, Science 2003, 300, 1127.

[19] The flow of a liquid on single-walled carbon nanotube bundles induces a voltage in the sample along the direction of the flow; see: S. Ghosh, A. K. Sood, N. Kumar, Science 2003, 299, 1042.

[20] N. N. Lavrik, P. G. Datskos, Appl. Phys. Lett. 2003, 82, 2697.

[21] S. S. Wong, J. D. Harper, P. T. Lansbury, C. M. Lieber, J. Am. Chem. Soc. 1998, 120, 603.

[22] J. K. Gimzewski, C. Joachim, R. R. Schlittler, V. Langlais, H. Tang, I. Johannsen, Science 1998, 281, 531.

[23] K. Keren, M. Krueger, R. Gilad, G. Ben-Yoseph, U. Sivan, E. Braun, Science 2002, 297, 72.

[24] S. Datta, W. Tian, S. Hong. R. Reifenberger, J. I. Henderson, C. P. Kubiak, Phys. Rev. Lett. 1997, 79, 2530.

[25] T. W. Kelley, E. L. Granstrom, C. D. Frisbie, Adv. Mater. 1999, 11, 261.

[26] J. Chen, M. A. Reed, A. M. Rawlett, J. M. Tour, Science 1999, $286,1550$.

[27] M. A. Reed, C. Zhou, C. J. Muller, T. P. Burgin, J. M. Tour, Science 1997, 278, 252.

[28] J. I. Martin, J. Nogués, Kai Liu, J. L. Vicent, Ivan K. Schuller, J. Magnetism and Magnetic Materials 2003, 256, 449.

[29] G. Maruccio, P.Visconti, S. D’Amico, P. Calogiuri, E. D'Amone, R. Cingolani, R. Rinaldi, Microelectronic Eng. 2003, 67-68, 838 .
[30] P. Visconti, G. Maruccio, E. D'Amone, A. Della Torre, A. Bramanti, R. Cingolani, R. Rinaldi, Mat. Sci. Eng. C-Bio S 2003, 23, 889 .

[31] G. Maruccio et al. unpublished results.

[32] A. Dodobalapur, H. E. Katz, L. Torsi, R. C. Haddon, Appl. Phys. Lett. 1996, 68, 1108.

[33] G. Gottarelli, S. Masiero, E. Mezzina, S. Pieraccini, J. P. Rabe, P. Samorì, G. P. Spada, Chem. Eur. J. 2000, 6, 3242.

[34] P. R. L. Malenfant, C. D. Dimitrakopoulos, J. D. Gelorme, L. L. Kosbar, T. O. Graham, A. Curioni, W. Andreoni, Appl. Phys. Lett. 2002, 80, 2517.

[35] M. J. Waner, M. Gilchrist, M. Schindler, M. Dantus, J. Phys. Chem. B 1998, 102, 1649.

[36] H. Nar, A. Messerschmitd, R. Huber, M. van de Kamp, G. W. Canters, J. Mol. Biol. 1991, 221, 765.

[37] E. P. Friis, J. E. T. Andersen, Y. I. Kharkats, A. M. Kuznetsov, R. J. Nichols, J. D. Zhang, J. Ulstrup, Proc. Natl. Acad. Sci. USA 1999, 96, 1379.

[38] J. J. Davis, D. Djuricic, K. K. W.Lo, E. N. K. Wallace, L. L. Wong, H. A. O. Hill, Faraday Discuss. 2000, 116, 15.

[39] J. R. Lakowicz, in Principles of Fluorescence Spectroscopy, 2nd ed., Plenum Press, New York 1999.

[40] S. J. Kroes, G. W. Canters, G. Gilardi, A. Van Hoek, and A. J. Visser, Biophys J. 1998, 75, 2441.

[41] A. Finazzi-Agrò, G. Rotilio, L. Avigliano, P. Guerrieri, V. Boffi, and B. Mondovì, Biochemistry 1970, 9, 2009.

[42] The unfolding process results in a large solvent exposure of hydrophobic residues that were shielded in the core of the protein in its native state.

[43] D. N. Blauch, J. M. Saveant, J. Am. Chem. Soc. 1992, 114, 3323.

[44] V. Frascerra, F. Calabi, G. Maruccio, P. P. Pompa, R. Cingolani, R. Rinaldi, Proc. 4th IEEE Conf. Nanotechnology 2004, Munich, Germany, 17-19/08/2004. 